\title{
Eine Beziehung zwischen den Algebren verallgemeinerter Distributionen von Colombeau und Ivanov
}

\author{
O. FÖLINCER
}

\begin{abstract}
We show a relation between an algebra of generalized distributions, which is generated by homogeneous distributions and defined by lvanov, and the algebra of generalized distributions defined by Colombeau.
\end{abstract}

Key words: Products of distributions, generalized distributions

AMS subject classification: $46 \mathrm{~F} 10$

0. Einfluhrung. Im Jahre 1954 bewies L. Schwartz in seiner Arbeit "Sur l' impossibilité .."[6], daß es nicht möglich ist, den Raum der Distributionen in eine Differentialalgebra einzubetten, die die Multiplikation der stetigen Funktionen verallgemeinert. Verschiedene Autoren haben später speziellere Produkte zwischen Distributionen definiert, etwa durch die Angabe von Kriterien für die Existenz des Produktes (Hörmander, Wladimirov, Ambrose u.a.) oder durch die Benutzung von speziellen Regularisierungen (Mikusinski, Hirata, Ogata u.a.). Siehe dazu die Arbeit von Oberguggenberger [4] und die in ihr zitierte Literatur. In der vorliegenden Arbeit soll ähnlich wie in derjenigen von Oberguggenberger die Beziehung eines von Ivanov [3] definierten Produktes homogener Distributionen zur Algebra von Colombeau untersucht werden. Dabei werden insbesondere Strukturaussagen über die Algebra von Colombeau gewonnen.

1. Die Algebra von Colombeau. Zur Definition der Algebra von Colombeau $G\left(R^{n}\right)$ benutzen wir die folgenden Indexmengen $A_{q}(q \in \mathbb{N})$ :

$$
A_{q}\left(\mathbb{R}^{n}\right)=\left\{\Phi \in C_{0}^{\infty}\left(\mathbb{R}^{n}\right)\left|\int \Phi(x) d x=1, \int \lambda^{\alpha} \cdot \Phi(x) d x=0 \forall \alpha \in Z_{+}^{n}, 1 \leq\right| \alpha \mid \leq q\right\} .
$$

Lemma 1: Seien $k$ linear unabhängige stetige Linearformen $L_{i}(i=1, \ldots, k)$ auf $C_{0}^{\infty}\left(\mathbb{R}^{n}\right)$ gegeben, $c=\left(c_{1}, \ldots, c_{k}\right) \in \mathbb{C}^{k}$ und $B=\left\{\Phi \in C_{0}^{\infty}\left(\mathbb{R}^{n}\right) \mid L_{i}(\Phi)=c_{i}\right.$ für alle $\left.i\right\}$. Dann ist $B \neq \Phi$.

Beweis: Wir wählen linear unabhängige Funktionen $\Phi_{i} \in C_{0}^{\infty}\left(\mathbb{R}^{n}\right)$ mit $L_{i}\left(\Phi_{i}\right) \neq 0$ so, daß die $L_{i}$, eingeschränkt auf $V=\operatorname{Lin}\left\{\Phi_{1}, \ldots, \Phi_{k}\right\}$, linear unabhängig sind. Folglich ist der Operator $L: V \rightarrow \mathbb{C}^{k}, L(\Phi)=\left(L_{1}(\Phi), \ldots, L_{k}(\Phi)\right)$, nach Konstruktion invertierbar. Sei $c \in \mathbb{C}^{k}$. Dann besitzt das Gleichungssystem $L(\Phi)=c(\Phi \in V)$ die Lösung $\Phi_{0}=L^{-1}(c), \Phi_{0} \in C_{0}^{\infty}\left(\mathbb{R}^{n}\right)$ und es gilt $\Phi_{\circ} \in B$

Folgerung 2: Es gilt $A_{q}\left(\mathbb{R}^{n}\right) \neq \Phi$ fur alle $q \in \mathbb{N}$.

Lemmal kann auch zur Konstruktion von Elementen aus $A_{q}$ benutzt werden, die weitere lineare Gleichungen erfiillen, sofern die Funktionale in diesen nur linear unabhängig sind. 
Für eine stetige Funktion $f: \mathbb{R}^{n} \rightarrow \mathbb{C}$ sei

$f_{\varepsilon}: \mathbb{R}^{n} \rightarrow \mathbb{C}, f_{\varepsilon}(x)=1 / \varepsilon n f(x / \varepsilon),\left(\tau_{y} f\right)(x)=f(x-y), \dot{f_{\varepsilon, x}}=\left(\tau_{x} f\right)_{\varepsilon}$.

Wir werden nun die grundlegende Définition der Colombeauschen Algebra geben.

Definition: Wir betrachten die Algebra

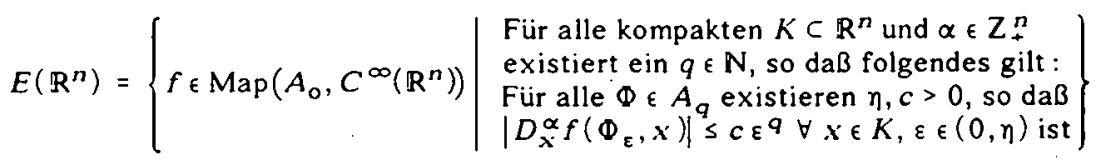

sowie das in ihr enthaltene Ideal

$$
N\left(\mathbb{R}^{n}\right)=\left\{\begin{array}{l|l}
f \in E\left(\mathbb{R}^{n}\right) & \begin{array}{l}
\text { Für alle kompakten } K \subset \mathbb{R}^{n} \text { und } \alpha \in Z_{+}^{n} \\
\text { existiert ein } l \in \mathbb{N}, \text { so daß folgendes gilt: } \\
\text { Füralle } q \in \mathbb{N}(q \geq l), \Phi \in A_{q} \text { gibt es } \eta, c>0, \text { so da } \beta \\
\left|D_{\lambda}^{\alpha} f\left(\Phi_{\varepsilon}, x\right)\right| \leq c \varepsilon q \forall x \in K, \varepsilon \in(0, \eta) \text { ist }
\end{array}
\end{array}\right\} .
$$

Dabei ist $D_{\lambda}^{\alpha} f$ die partielle Ableitung der glatten Funktion $f\left(\Phi_{\varepsilon}, \cdot\right): \mathbb{R}^{n \prime} \rightarrow \mathbb{C}$ für festes $\Phi_{\varepsilon}$. Die Quotientenalgebra $G\left(\mathbb{R}^{n}\right)=E\left(\mathbb{R}^{n}\right) / N\left(\mathbb{R}^{n}\right)$ wird als Algebra von Colombeau bezeichnet. Die Elemente von ihr werden verallgemeinerte Distributionen genannt.

Der Raum der Distributionen $D^{\prime}\left(\mathbb{R}^{n}\right)$ ist vermöge $T(\Phi, x)=\langle T, \tau, \Phi\rangle$ in $G\left(\mathbb{R}^{n}\right)$ eingebettet und $C^{\infty}\left(\mathbb{R}^{n}\right)$ ist eine Differential-Unteralgebra von $G\left(\mathbb{R}^{n}\right)$. Eine ausfuhrliche Beschreibung der Algebra $G\left(\mathbb{R}^{n}\right)$ findet man in $[2,5]$. Im weiteren werden die verallgemeinerten Distributionen und ihre Repräsentanten miteinander identifiziert. Auf Grund des Resultates von Schwartz [6] ist klar, daß $C\left(\mathbb{R}^{n}\right)$ keine Unteralgebra von $G\left(\mathbb{R}^{n}\right)$ sein kann. Colombeau [2] stellt eine Beziehung zwischen diesen Algebren mit Hilfe des Begriffes der assoziierten Distribution her. Eine Distribution $T \in D^{\prime}\left(R^{n}\right)$ heißt assoziiert zu einer verallgemeinerten Distribution $F \in \mathcal{G}\left(\mathrm{R}^{n}\right)$, wenn für ein $q \in \mathbb{N}$ die Beziehung

$$
\lim _{\varepsilon \rightarrow 0} \int F\left(\Phi_{\varepsilon}, x\right) \psi(x) d x=\langle T, \psi\rangle \text { für alle } \Phi \in A_{q}\left(\mathbb{R}^{n}\right), \psi \in D\left(\mathbb{R}^{n}\right)
$$

gilt. Damit ist $T$ eindeutig und repräsentantenunabhängig definiert. Colombeau bewies in [2], daB für eine Distribution $T$ die Beziehung $\lim _{\varepsilon \rightarrow 0} \int T\left(\Phi_{\varepsilon}, x\right) \psi(x) d x=\langle T, \psi\rangle$ gilt. Also ist jede Distribution zu sich selbst assoziiert.

2. Homogene Distributionen in $G(R)$. Ivanov [3] definierte eine Multiplikation in einem von homogenen Distributionen aufgespannten Unterraum von $D^{\prime}(R)$. Dabei werden insbesondere Distributionen mit gleichem singulären Träger miteinander multipliziert. Eine Distribution $T \in D^{\prime}\left(\mathbb{R}^{n}\right)$ heißt homogen vom Grad $k \in \mathbb{N}$, wenn $\left\langle T, \Psi_{1 / t}\right\rangle=t^{k}\langle T, \psi\rangle$ für alle $\psi \in D\left(\mathbb{R}^{n}\right)$ gilt. Sei $H_{0}(\mathbb{R})$ der von

$$
\delta^{(n-1)}=\frac{d^{n-1}}{d x^{n-1}} \delta, \text { v.p. } \dot{x}^{-m}=\frac{(-1)^{m-1}}{(m-1) !} \frac{d^{m}}{d x^{m}} \ln |x| \quad(m, n \in \mathbf{N})
$$

und den komplexen Konstanten aufgespannte Unterraum von $D^{\prime}(\mathbb{R})$ und $H(\mathbb{R})$ die von $H_{\mathrm{o}}\left(\mathbb{R}^{\prime \prime}\right)$ erzeugte freie Algebra. $H_{\mathrm{o}}(\mathbb{R})$ ist als Unterraum des Raumes der Distributionen in $G(R)$ eingebettet und es gilt das folgende

Theorem 3: Die Algebra $H(\mathbb{R})$ ist in $G(\mathbb{R})$ als Unteralgebra eingebettet. 
Beweis: Es genügt zu zeigen, daß $H(\mathbb{R}) \cap N(\mathbb{R})=\{0\}$ ist. Sei $S \in H(\mathbb{R}), S \neq 0$, ein Monom der Homogenität $k \in \mathbb{N}$ (unter Homogenität eines Monoms verstehen wir die Summe der Homogenitäten der einzelnen Distributionen), dàs heißt

$$
S=\prod_{i=1}^{n} T_{i}, T_{i} \in\left\{\delta^{(p-1)}, \text { v.p. } x^{\cdots m} \mid m, p \in N\right\}, \operatorname{hom}\left(T_{i}\right)=k_{i}, k=\sum_{i=1}^{n} k_{i} .
$$

Dann gilt bei $x=0$ die Beziehung

$$
\begin{aligned}
\left|S\left(\Phi_{\varepsilon}, 0\right)\right| & =\left|\left\langle T_{1}, \Phi_{\varepsilon}\right\rangle \ldots\left\langle T_{n}, \Phi_{\varepsilon}\right\rangle\right| \\
& =\left|\varepsilon^{-k_{1}}\left\langle T_{1}, \Phi\right\rangle \ldots \varepsilon^{-k_{n}}\left\langle T_{n}, \Phi\right\rangle\right|=\varepsilon^{-k}\left|\left\langle T_{1}, \Phi\right\rangle \ldots\left\langle T_{n}, \Phi\right\rangle\right|=c \varepsilon^{-k}
\end{aligned}
$$

unabhängig davon, ob $\Phi \in A_{q}$ oder $\Phi \notin A_{q}$ ist. Also gilt für ein beliebiges Monom $S$ die Beziehung $S \& N(\mathbb{R})$. Sei nun $S \in H(\mathbb{R})$ beliebig gewählt, das heißt

$$
S=\sum_{i=1}^{n} c_{i} S_{i} \quad\left(0 \neq c_{i} \in \mathbb{C} ; S_{i} \in H(\mathbb{R}) \text { ein Monom }\right) .
$$

Summen von Monomen unterschiedlicher Homogenität sind keine Elemente von $N(\mathbb{R})$ auf Grund des unterschiedlichen Verhaltens fur $\varepsilon \rightarrow 0$. Summen von Monomen gleicher Homogenität ergeben wieder verallgemeinerte Distributionen, die die Gleichung (1) erfüllen. Eine solche Summe ist nur dann Element von $N(\mathbb{R})$, wenn ein $q$ existiert, so daß in (1) $c=0$ für alle $\Phi \in A_{q}$ ist. Wir zeigen, daß fur alle $q \in \mathbb{N}$ eine Funktion $\Phi \in A_{q}$ existiert, für die $c \neq 0$ ist. Seien dazu die $S_{i}$ in (2) Monome der Homogenität $k \in \mathbb{N}$ und $q \in \mathbb{N}$ fest. Sei weiterhin $\mathbf{T}=\left\{T_{1}, \ldots, T_{r}, T_{r+1}, \ldots, T_{s} \mid r, s \in \mathbb{N}\right\} \subset H_{\mathrm{o}}(\mathbb{R})$ die Menge der Distributionen, aus denen sich $S$ zusammensetzt, so daB $S_{1}=T_{1} \ldots T_{r}$ ist, und die $S_{i}$ seien für $i \neq 1$ Produkte von Distributionen aus $\mathbf{T}$; jedoch nicht gleich $S_{1}$. Da die Elemente von $\mathbf{T}$ sowohl voneinander als auch von den definierenden Funktionalen der Mengen $A_{q}$ linear unabhängig sind, folgt aus Lemma 1 die Existenz eines $\Phi \in A_{q}$ mit $\left\langle T_{1}, \Phi\right\rangle=\ldots=\left\langle T_{r}, \Phi\right\rangle=1$ und $\left\langle T_{r+1}, \Phi\right\rangle=\ldots=\left\langle T_{s}, \Phi\right\rangle=0$. Dies ist die gesuchte Funktion $\Phi \in A_{q}$

Theorem 4: Ein Element $S \in H(\mathbb{R})$ besitzt genau dann eine assoziierte Distribution, wenn $S \in H_{0}(\mathbb{R})$ ist.

Beweis: Es genügt zu zeigen, daß $S \in H(\mathbb{R}) \backslash H_{\mathrm{o}}(\mathbb{R})$ keine assoziierte Distribution besitzt. Sei $S$ zunächst ein Monom der Homogenität $p \in \mathbb{N}$. Dann gilt

$$
T=\left(\prod_{i=1}^{k} T_{i}\right)\left(\prod_{j=1}^{l} S_{j}\right)(k, l \in \mathbb{N}, k+l>1), T_{i}=\delta^{\left(n_{i}-1\right)}, S_{j}=\text { v.p. } x^{-m_{j}}\left(n_{j}, m_{j} \in \mathbb{N}\right) .
$$

Aus $k+1>1$ folgt $\rho>1$. Wir berechnen zunächst die Bilder der Einbettungen von $\delta^{(n-1)}$ und v.p. $x^{-m}$ in $G(\mathbb{R})$. Dabei ist

$$
\begin{aligned}
& \left\langle\delta^{(n-1)},\left(\tau_{x} \Phi\right)_{\varepsilon}\right\rangle=(-1)^{n-1} \varepsilon^{-n} \Phi^{(n-1)}(-x / \varepsilon) \\
& \left\langle\text { v.p. } x^{-m},\left(\tau_{x} \Phi\right)_{\varepsilon}\right\rangle=(-1)^{m} \varepsilon^{-m} \int \ln |x+\varepsilon \mu| \Phi^{(m)}(\mu) d \mu .
\end{aligned}
$$

Wir betrachten nun $\psi \in C_{0}^{\infty}(\mathbb{R})$ mit $\psi(0) \neq 0$ und $\Phi \in A_{q}$. Es ist dann

$$
\begin{aligned}
& \lim _{\varepsilon \rightarrow 0} \int S\left(\Phi_{\varepsilon}, x\right) \psi(x) d x \\
& \quad=\lim _{\varepsilon \rightarrow 0} \int\left(\prod_{j=1}^{k}\left\langle\delta^{\left(n_{j}-1\right)} ;\left(\tau_{x} \Phi\right)_{\varepsilon}\right\rangle\right)\left(\prod_{j=1}^{l}\left\langle v: p_{.} x^{-m},\left(\tau_{x} \Phi\right)_{\varepsilon}\right\rangle\right) \psi(x) d x \\
& \quad=\lim _{\varepsilon \rightarrow 0} \int\left(\prod_{i=1}^{k}(-1)^{n_{j}-1} \varepsilon^{-n_{i}} \Phi^{\left(n_{j}-1\right)}(-x / \varepsilon)\right)\left(\prod_{j=1}^{l}(-1)^{m_{j}} \int \ln \left|x+\varepsilon \mu_{j}\right| \Phi^{\left(m_{j}\right)}\left(\mu_{j}\right)\right) \psi(x) d x
\end{aligned}
$$




$$
=\lim _{\varepsilon \rightarrow 0} \varepsilon^{-M-N+1}(-1)^{M+N} \int\left(\prod_{i=1}^{k} \Phi^{\left(n_{i}-1\right)}(-x)\right)\left(\prod_{j=1}^{1} \int \ln \left(\varepsilon\left|x+\varepsilon \mu_{j}\right|\right) \Phi^{\left(m_{j}\right)}\left(\mu_{j}\right)\right) \Psi(\varepsilon x) d x .
$$

Dabei gilt $N=\sum_{i=1}^{k} n_{i}, M=\sum_{j=1}^{1}\left(m_{j}-1\right)$ und $p=M+N>1$. Wir erhalten Summen von Termen der Form

$$
c(\ln \varepsilon)^{r} \varepsilon^{-p+1} \int\left(\prod_{i=1}^{k} \Phi^{\left(n_{i}-1\right)}(-x)\right)\left(\prod_{j=1}^{l} \int \ln \left|x+\mu_{j}\right| \Phi^{\left(m_{j}\right)}\left(\mu_{j}\right) d \mu_{j}\right) \psi(\varepsilon x) d x,
$$

wobei $c$ nur kombinatorische Größen in $k$ und $l$ enthält. Für $\varepsilon \rightarrow 0$ verhalten sich die Terme in (5) wie $O\left((\ln \varepsilon)^{r^{-p+1}}\right)$, können in der Summe also nur verschwinden, wenn jeder einzelne Summand verschwindet. Es genügt also zunächst, einen dieser Terme zu betrachten. Da $p>1$ ist, kann der Term in (5) nur dann verschwinden, wenn die Integrale

$$
I(\Phi)=\int\left(\prod_{i=1}^{k} \Phi^{\left(n_{i}-1\right)}(-x)\right)\left(\prod_{j=1}^{l} \int \ln \left|x+\mu_{j}\right| \Phi^{\left(n_{j}\right)}\left(\mu_{j}\right) d \mu_{j}\right) \psi(\varepsilon x) d x
$$

betrachtet als nichtlineare Funktionale auf $A_{q}$, für alle $q \in \mathbb{N}$ identisch verschwinden. Notwendig dafür ist, daß für $q \in \mathbb{N}$ und für alle $\varphi, \Phi \in A_{q}$ die Gateaux-Ableitung $\left(D_{\mathrm{G}} I(\Phi)\right)(\varphi)$ $=d /\left.d t I(\Phi+t \varphi)\right|_{t=0}$ von $I$ verschwindet. Dies liefert ein Funktional, angewendet auf $\varphi$, das linear unabhängig von den definierenden Funktionalen der Menge $A_{q}$ ist. Mit Lemma 1 können wir nun schließen, daß für alle $q \in \mathbb{N}$ ein $\varphi \in A_{q} \operatorname{mit}\left(D_{\mathrm{G}} I(\Phi)\right)(\varphi)=1$ existiert. Also verschwindet $I(\Phi)$ nicht identisch und es existiert ein $\Phi \in A_{q}$, für das der Grenzwert von (5) für $\varepsilon \rightarrow 0$ nicht existiert, das heißt $S$ besitzt keine assoziierte Distribution. Ein allgemeines Element von $H(\mathbb{R}) \backslash H_{\mathrm{o}}(\mathbb{R})$ hat die Form

$$
S=\sum_{i=1}^{n} c_{i} S_{i}\left(c_{i} \in \mathbb{C}, S_{i} \in H(\mathbb{R})\right),
$$

wobei $S_{i} H_{0}(\mathbb{R})$ für mindestens ein $i$ ist. Summen von Monomen verschiedener Homogenität können furr $\varepsilon \rightarrow 0$ auf Grund ihres unterschiedlichen Grenzwertverhaltens nicht verschwinden. Für Summen von Monomen gleicher Homogenität, von denen keines Element von $H_{\mathrm{o}}(\mathbb{R})$ ist, kann wie für ein Monom argumentiert werden. Es bleibt der Fall zu betrachten, daß $S=T+R$ ist, wobei $T \in H_{0}(\mathrm{R})$ natiurlich eine assoziierte Distribution besitzt und keiner der Summanden von $\mathrm{R}$ ein Element aus $H_{\mathrm{o}}(\mathbb{R})$ ist. Besäße $S$ in diesem Fall eine assoziierte Distribution, so auch $R=S-T$. Das steht aber im Widerspruch zu der oben gezeigten Behauptung. Also besitzt $S$ keine assoziierte Distribution

3. Die Algebra von Ivanov. Ivanov definiert in [3] eine andere Algebra iber $H_{\mathrm{o}}(\mathbb{R})$. Er betrachtet dazu den Vektorraum $F_{0}=\operatorname{lin}\left\{z^{-n}, \bar{z}^{-n} \mid n \in \mathbf{N}\right\}$ der in der oberen Halbebene harmonischen Funktionen $z^{-n}, \bar{z}^{-n}$ und nutzt den Fakt, daß dieser Raum isomorph $z \mathrm{u} H_{\mathrm{o}}(\mathbb{R})$ ist. Die Isomorphie wird durch die Beziehungen

$$
\text { v.p. } x^{-m} \Leftrightarrow 1 / 2\left(z^{-m}+\bar{z}^{-m}\right) \text { und }(-1)^{n \cdot 1 /(n-1) \delta^{(n-1)} \Leftrightarrow 1 / 2 \pi \mathrm{i}}\left(z^{-n}-\bar{z}^{-n}\right)
$$

angegeben. Sei $F$ die von $F_{\mathrm{o}}$ erzeugte Funktionalgebra. Durch Ausdehnung des angegebenen Isomorphismus wird uber $H_{0}(\mathrm{R})$ die Struktur einer Algebra $H_{1}(\mathbb{R})$ definiert. In dieser Algebra gilt zum Beispiel die Beziehung

$$
\delta \text { v.p. } x^{-1} \Leftrightarrow 1 / 2 \pi \mathrm{i}\left(z^{-1}-\bar{z}^{-1}\right) 1 / 2\left(z^{-1}+\bar{z}^{-1}\right)=1 / 4 \pi \mathrm{i}\left(z^{-2}-\bar{z}^{-2}\right) \Leftrightarrow-1 / 2 \delta^{(1)},
$$

folglich ist $\delta$ v.p. $x^{1}=-1 / 2 \delta^{(1)}$ in $H_{1}(\mathbb{R})$. Daraus erhält man mit Theorem 3 , daß $H_{1}(\mathbb{R})$ keine Unteralgebra von $H_{0}(\mathbb{R})$ ist, da in $H_{0}(\mathbb{R})$ die Beziehung $\delta$ v.p. $x^{-1} \neq \delta^{(1)}$ gilt. Der Beweis von 
Theorem 4 zeigt weiterhin, daß $\delta$ v.p. $x^{-1}$ keine assoziierte Distribution besitzt, insbesondere auch nicht $z u \delta^{(1)}$ assoziiert ist. Für die Algebra $H_{1}(\mathbb{R})$ gilt nun der folgende

Satz 5: Der Vektorraum $H_{1}(\mathbb{R})$ ist als Unterraum in $G(\mathbb{R})$ eingebettet. Er ist keine Unteralgebra von $G(\mathrm{R})$.

Beweis : Ein beliebiges Element $S \in H_{1}(\mathbb{R})$ der Homogenität $k$ hat die Poisson-Darstellung $S=\sum_{i+j=k} a_{i j} z^{-i} \bar{z}^{-j}, a_{i j} \in \mathbb{C}$. Da

$$
2 z^{-1}=\left(z^{-1}+\bar{z}^{-1}\right)+\left(z^{-1}-\bar{z}^{-1}\right) \text { und } 2 \bar{z}^{-1}=\left(z^{-1}+\bar{z}^{-1}\right)-\left(z^{-1}-\bar{z}^{-1}\right)
$$

gilt, kann $S$ als Summe von Produkten von Elementen aus $H_{\mathrm{o}}(\mathbb{R})$ dargestellt werden, das heißt $H_{1}(\mathbb{R})$ ist in $H(\mathbb{R})$ als Unterraum eingebettet. Da letzterer nach Theorem 3 in $G(\mathbb{R})$ eingebettet ist, gilt dies auch für $H_{1}(\mathbb{R})$. Der Rest der Behauptung ergibt sich aus der Bemerkung vor dem Satz

Zur Definition einer Multiplikation in $H_{0}(\mathbb{R})$ benutzt Ivanov eine multiplikative Regularisierung. Wir geben deshalb die folgende

Definition: Sei $G$ eine Algebra uiber $H_{\mathrm{o}}(\mathbb{R})$. Eine lineare Abbildung $\lambda: G \rightarrow H_{\mathrm{o}}(\mathbb{R})$ heißt Regularisierung, falls folgendes gilt:

1. Die Einschränkung von $\lambda$ auf $H_{0}(\mathbb{R})$ ist die identische Abbildung.

2. $\operatorname{Ker} \lambda \subset\{T \in G \mid T$ ist in 0 konzentriert $\}$.

Eine Regularisierung heißt multiplikativ, falls $\lambda(S T)=\lambda(\lambda(S) \lambda(T))$ für alle $S, T \in G$ gilt.

Ist $\lambda$ eine multiplikative Regularisierung, so kann wie folgt eine Multiplikation $\circ$ in $H_{\mathrm{o}}(\mathbb{R})$ definiert werden:

$\circ: H_{\mathrm{o}}(\mathbb{R}) \times H_{\mathrm{o}}(\mathbb{R}) \rightarrow H_{\mathrm{o}}(\mathbb{R}), S \circ T=\lambda(S T)$.

Ivanov zeigt nun in [3] den folgenden

Satz 6 : Es existiert genau eine multiplikative Regularisierung $\lambda_{0}: H_{1}(\mathbb{R}) \rightarrow H_{0}(\mathbb{R})$, so daB die mit ihrer Hilfe definierte Multiplikation o in $H_{\mathrm{o}}(\mathbb{R})$ die Struktur einer assoziativen kommutativen reellen Differentialalgebra bezüglich $d / d x$ erzeugt ("reell" bedeutet, daß reellwertige Testfunktionen in $\mathbb{R}$ abgebildet werden). Dabei gilt

$$
H_{1}(\mathrm{R}) \stackrel{\mathrm{pr}}{\longrightarrow} H_{1}(\mathrm{R}) / l \stackrel{j}{\longrightarrow} H_{0}(\mathrm{R}), \lambda_{0}=j \circ \mathrm{pr},
$$

wobei pr die Projektion auf die Faktoralgebra $H_{1}(\mathbb{R}) / I, j$ ein kanonischer Isomorphismus und $I$ das von $\delta^{2}$ erzeugte Hauptideal in $H_{1}(\mathbb{R})$ ist .

Der Beweis ergibt sich aus der Folgerung nach Theorem 9 in [3]

Nach Theorem 4 ist klar, daß die Elemente des Ideals $I$ keine assoziierten Distributionen besitzen. Unser $\mathrm{Ziel}$ ist es, eine Regularisierung $x: H(\mathbf{R}) \rightarrow H_{\mathrm{o}}(\mathbb{R})$ zu definieren. "Die Idee dazu ist einfach: Für eine beliebige kommutative Algebra uber einem .Vektorraum existiert ein kanonischer Homomorphismus $\mu$ der freien kommutativen Algebra in diese Algebra, der eindeutig durch die Bedingung gegeben ist, daß seine Einschränkung auf den Vektorraum die Identität ist. Sei $\mu: H(\mathbb{R}) \rightarrow H_{1}(\mathbb{R})$ dieser Homomorphismus. 
Satz 7: Die Abbildung $x=\lambda_{0} \circ \mu: H(\mathbb{R}) \rightarrow H_{0}(\mathbb{R})$ ist eine multiplikative Regularisierung. Die Elemente von $\operatorname{Ker} x$ besitzen keine assoziierten Distributionen.

Beweis : Die Abbildung $x$ ist nach Konstruktion, eingeschränkt auf $H_{0}(\mathbb{R})$, die Identität. Wir benutzen jetzt, daB die Elemente von $G(R)$ auf offene Teilmengen von $\mathbb{R}$ eingeschränkt werden können [2].Wir betrachten die Einschränkung $x_{0}: H(\mathbb{R} \backslash\{0\}) \rightarrow H_{0}^{\wedge}(\mathbb{R} \backslash\{0\})$ von $x$. Auf $\mathbb{R} \backslash\{0\}$ sind die Elemente von $H(\mathbb{R})$ glatte Funktionen, deren Gesamtheit in $G(\mathbb{R})$ als Differential-Unteralgebra eingebettet ist [2], und es gilt

$$
H(\mathbb{R} \backslash\{0\})=\operatorname{Lin}\left\{f_{m} \mid f_{m}(x)=x^{-m}, x \in \mathbb{R}, m \in \mathbb{N}\right\}=H_{o}(\mathbb{R} \backslash\{0\}) .
$$

Hierauf ist $x_{0}$ nach Konstruktion die Identität, also liegen diejenigen Elemente von $H(\mathbb{R})$, die auf $R \backslash\{0\}$ nicht verschwinden, nicht in Ker $x$. Folglich ist $x$ eine Regularisierung.

Für den Beweis des zweiten Teils der Behauptung müssen wir Ker $x$ genauer charakterisieren. Dabei gilt $\operatorname{Ker} x=\operatorname{Lin}\left\{\operatorname{Ker} \mu+\mu^{11} \operatorname{Ker} \lambda_{0}\right\}=\operatorname{Lin}\left\{\operatorname{Ker} \mu+\delta^{2} H(\mathbb{R})\right\}$. Aus rein algebraischen Gründen ist klar, daB Ker $\mu$ die lineare Hülle all derjenigen Hauptideale ist, die von einem beliebigen Element der Form

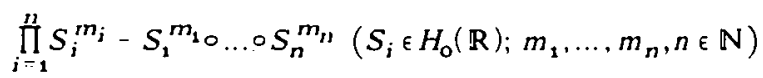

erzeugt werden: Dabei bezeichnet o die Multiplikation in $H(\mathbb{R})$ und $\Pi$ die Operation in $H_{1}(\mathbb{R})$, wobei das Ergebnis als Element von $H(\mathbb{R})$ verstanden wird. Sowohl für $\sum_{i=1}^{n} m_{i}=0$ als auch für $\sum_{i=1}^{n} m_{i}=1$ ist die Differenz in (7) gleich Null. Für $\sum_{j=1}^{n} m_{i}>1$ ist die Differenz entweder Null, oder das von ihr erzeugte Hauptideal ist eine Teilmenge von $H(\mathbb{R}) \backslash H_{\mathrm{o}}(\mathbb{R})$. Da auch $\delta^{2} H(\mathbb{R})$ Teilmenge von $H(\mathbb{R}) \backslash H_{0}(\mathbb{R})$ ist, gilt die Behauptung nach Theorem 4

Damit ist gezeigt, daß bei der Definition der Multiplikation in $H_{\mathrm{o}}(\mathbb{R})$ mit Hilfe von $x$ oder $\lambda_{0}$ genau diejenigen Elemente der über $H_{0}(\mathbb{R})$ betrachteten Algebra wegfaktorisiert werden, die keine assoziierten Distributionen besitzen.

\section{LITERATUR}

[1] ColombeaU, J.F.: A Multiplication of Distributions. J. Math. Anal. Appl. 94 (1983), $96-115$.

[2] COLOMBEAU, J.F.: New Generalized Functions and Multiplication of Distributions. Amsterdam: North Holland Publ. Comp. 1984.

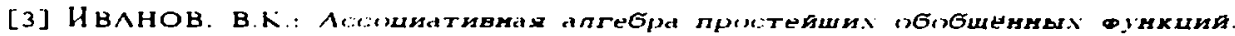
Сиб. мат. ж. 20 (1979), 731 - 740.

[5] ObergugGenberger, M.: Products of distributions. J. reine angew. Math. 365 (1986), 1 - 11 .

[5] ROSINGER, E.E.: Generalized Solutions of Nonlinear Partial Differential Equations. Amsterdam: North - Holland Publ. Comp. 1987.

[6] SCHWARTZ, L: Sur $r$ impossibilité de la multiplication des distributions. C.R. Akad. Sci. Paris (Série A) 239 (1954), $847-848$.

Received 19.09. 1989; in revised form 25.07.1990

Author's address:

Olaf Föllinger

Institut fir Reine Mathematik der Humboldt-Universität

Unter den Linden 6

D (Ost) - 1086 Berlin 